\title{
Reflexiones acerca de la asexualidad a partir de dos experiencias clínicas en el CRAm
}

\section{Reflections on asexuality based on two clinical experiences in CRAm}

\author{
Gonzalo Iván Gelpi \\ Facultad de Psicología \\ Universidad de la República, Uruguay. \\ ggelpi@psico.edu.uy \\ https://orcid.org/0000-0002-5778-8818 \\ Nahuel Suñol \\ Facultad de Psicología \\ Universidad de la República, Uruguay. \\ nahuesun978@gmail.com \\ https://orcid.org/0000-0001-5094-7049
}

\section{Resumen}

En este artículo se pretende reflexionar desde una mirada crítica sobre la asexualidad. La selección de la temática se relaciona con la necesidad de visibilizar a una comunidad que muchas veces es negada. Generalmente la asexualidad ocupa un sitio periférico dentro de la agenda de investigación y de las discusiones científicas dentro del campo de la sexualidad humana y de los estudios de género, lo que genera una baja producción académica en español en esta materia. Para cumplir con este objetivo, se realiza una síntesis relativa al estado del arte sobre la temática y se propone una articulación teórico-práctica en base a dos experiencias clínicas con usuarios autoidentificados como asexuales en el marco de la atención psicológica que se ofrece en el Centro de Referencia Amigable (CRAm) en Uruguay. Este trabajo, lejos de buscar patologizar a la asexualidad, pretende ser un punto de partida para la reflexión, la crítica y la revisión de las distintas concepciones, enfoques y abordajes acerca de la asexualidad, con el fin de motivar la formulación de nuevas interrogantes y promover un aumento de producción de conocimiento sobre el fenómeno.

Palabras clave: Psicología; Sexualidad; Diversidad sexual; Asexualidad. 


\begin{abstract}
This article aims to reflect from a critical perspective on asexuality. The selection of the theme is related to the need to visualize a community that is often denied. Asexuality generally occupies a peripheral place within the research agenda and scientific discussions within the field of human sexuality and gender studies, which generates a low academic production in Spanish in this area. To meet this objective, a synthesis regarding the state of the art on the subject is carried out and a theoretical-practical articulation is proposed based on two clinical experiences with users selfidentified as asexual within the framework of the psychological care offered at the Center of Friendly Reference (CRAm) in Uruguay. This work, far from seeking to pathologize asexuality, aims to be a starting point for reflection, criticism and review of the different conceptions, approaches and approaches about asexuality, in order to motivate the formulation of new questions and promote an increase in the production of knowledge about the phenomenon.
\end{abstract}

Key words: Psychology; Sexuality; Sexual diversity; Asexuality. 


\section{1 ¿Qué es el Centro de Referencia Amigable (CRAm)?}

El Centro de Referencia Amigable $(\mathrm{CRAm})^{1}$ es un servicio universitario de atención psicológica dirigido a población LGBT+ residente en Uruguay, que existe desde el año 2014, a partir de un convenio entre la Facultad de Psicología (UdelaR) y el Ministerio de Desarrollo Social (MIDES). EI objetivo del servicio es brindar un espacio de consulta, orientación y/o atención psicológica a la población LGBT+ desde un paradigma de derechos humanos que contemple la perspectiva de género y diversidad, lo cual garantiza el reconocimiento de la orientación sexual, identidad de género y expresión de género de los usuarios y usuarias en los espacios de consulta. Las intervenciones psicosociales que se promueven en CRAm son sensibles al género y en el sentido más amplio pretenden ser transformadoras de género. Se destaca que en el CRAm se realizan las tres funciones universitarias: enseñanza, investigación y extensión. Asimismo, se cumplen todas las normativas éticas inherentes a la atención e investigación con seres humanos en Uruguay ${ }^{2}$. Actualmente se ofrecen tres prestaciones: consultas únicas de apoyo legal y/o psicológico, psicoterapias individuales focales y dispositivos grupales no terapéuticos de prevención primaria y secundaria. La atención se brinda presencialmente y también a distancia. En promedio, entre las dos primeras prestaciones mencionadas, se atienden 60 consultas anuales. El servicio busca fortalecer la capacidad de dar respuesta institucional, articulando la atención en salud mental a la población LGBT+ y el desarrollo de estrategias de prevención en salud, promoción de derechos, producción de conocimiento y formación de recursos humanos.

\section{Dos experiencias de atención psicológica a personas autoidentificadas como asexuales en el CRAm}

En esta oportunidad, se pretende reflexionar sobre la asexualidad, en base a dos experiencias clínicas de procesos psicoterapéuticos con personas autoidentificadas como asexuales. La principal técnica empleada en el trabajo directo con estas personas fue la entrevista clínica en profundidad, complementada por la Línea de Vida Modificada ${ }^{3}$. Para cumplir con tal fin, se articula la teoría con viñetas clínicas especialmente seleccionadas para esta ocasión. Se resalta que se parte de un total respeto hacia la asexualidad, hacia la historia de vida de estos consultantes y no se pretende bajo ningún concepto operar desde un paradigma patologizante. El ejercicio que nos proponemos es hacer un acercamiento preponderantemente desde la psicología clínica y visibilizar la temática, problematizarla, proponer de cierta forma una articulación teórica novedosa y actual, reconociendo que se trata de una casuística muy pequeña, lo que configura una limitante, por lo cual, todas las

\footnotetext{
${ }^{1}$ Por más información pueden acceder a: https://psico.edu.uy/novedades/portada-de-sitio/centro-de-referenciaamigable-cram.

2 Dependiendo de la edad, para recibir atención, los usuarios y usuarias deben firmar un consentimiento y/o asentimiento informado. A su vez, en la primera entrevista, se les entrega una hoja de información con todos los detalles sobre el funcionamiento del servicio. Se aclara que, en los casos que las personas no desean ceder sus datos con fines de investigación, igualmente se les brinda atención psicológica.

${ }^{3}$ Por más información sobre la técnica se puede consultar el capítulo 6 de la Guía Salud y Diversidad Sexual elaborada por López, Forrisi y Gelpi (2015).
} 
conclusiones son parciales y no tienen la intención de ser aplicadas para todas las experiencias de asexualidad. Sin embargo, también se debe destacar que ambos usuarios, tuvieron más de 30 consultas en nuestro servicio, lo que permite pensar que hay un conocimiento relativamente profundo acerca de las conflictivas que presentaban estas personas, así como también, sobre sus características de personalidad y sus modos de producción de subjetividad.

Por un lado, está el caso de $\mathbf{Q}$, una mujer cisgénero, de 33 años, de nacionalidad uruguaya, trabajadora independiente, autoidentificada como asexual, que consulta por sentir una confusión respecto a su identidad y orientación sexual, ella describe sentir un "torbellino en relación a su identidad y un conflicto con la autopercepción de su imagen corporal". Q descubrió la categoría asexual recientemente, considera que dicha categoría la describe. Desde que tiene recuerdos se sintió así, "sin deseo ni atracción física/sexual o emocional hacia alguien". En el proceso de consulta, la usuaria narra haber sido víctima de abuso sexual en la infancia, alrededor de los 10 o 11 años, no lo recuerda bien y su familia descreyó el relato. A medida que avanza el análisis, ciertos recuerdos comienzan a desbloquearse, logra asociar que su rechazo por sus senos podría relacionarse con el tipo de prácticas que acontecían durante los abusos. A su vez, su autoidentificación como asexual comenzó a problematizarse en las consultas, ya no era algo indudable, una total certeza, sino que surgieron preguntas. Más adelante, ella trabaja ciertas dificultades vinculares principalmente con varones, relacionarse con ellos le genera "miedo, inseguridad y desconfianza", siente la falta de deseo sexual. Nos comenta que una vez probó estar con un varón, intentó salir, besarse, pero no llegó a materializar una práctica sexual genital porque todo el encuentro lo describe como "un embole". La usuaria trae su asexualidad asociada a un malestar psíquico, le produce angustia, se compara con el resto y cree que algo no está bien en ella, sin embargo, al mismo tiempo, su núcleo de malestar no se relaciona con sus propias expectativas y deseos en materia de sexualidad sino que más bien se vincula con las expectativas y deseos que tienen los demás sobre ella, es decir, sus familiares directos, sus mejores amigas y amigos, entre otros actores cercanos. Más adelante, $\mathbf{Q}$ plantea haberse masturbado pocas veces durante su vida, reconoce que sintió placer, pero este tipo de prácticas no le interesaron lo suficiente como para seguir realizándolas habitualmente. Es posible afirmar que, la usuaria, tiene una vida autoerótica con interrupciones y su asexualidad responde especialmente a la dimensión vincular de la sexualidad. Uno de los logros terapéuticos con la usuaria fue trabajar sobre su imagen, que se pueda apropiar subjetivamente de su cuerpo y su corporalidad, esto trajo consigo cambios en su presentación cotidiana, transitó de un aspecto predominantemente andrógino a uno que podría interpretarse como femenino. Esto no es menor porque como se sabe, la expresión de género es parte del concepto de sexualidad. Este cambio la hizo sentirse mejor y su propio entorno lo notó.

Por otro lado, está el caso de $\mathbf{Y}$, un varón trans, de 17 años, de nacionalidad uruguaya, estudiante de Enseñanza Media, autoidentificado como asexual, que consulta para poder acceder a un tratamiento de hormonización porque siente una insatisfacción con su cuerpo que impacta negativamente en su autoestima. El usuario cree que la categoría asexual es la que mejor lo describe, pero no logra contestar la pregunta de qué significa la asexualidad para él, la evade. Sin embargo, responde que "el prefijo a adelante indica no, así que la asexualidad sería no tener sexualidad". Durante el proceso de consulta el usuario también narra haber sido víctima de abusos sexuales en la infancia, el caso llegó a la justicia y él acabó siendo institucionalizado por un par de años, pero insiste en que ya no quiere hablar de eso porque lo trabajó en su anterior psicoterapia, aunque luego terminó revirtiendo su postura. A medida que avanza el análisis, el proceso con $\mathbf{Y}$ se caracteriza por la usual presencia de metáforas traídas por el mismo en cada consulta. En una sesión, aproximadamente dos meses después de haber iniciado el tratamiento, el usuario decide colocar su 
sexualidad en el centro del análisis. Plantea que "me cuesta expresar emociones, tengo interés de conocer a alguien, pero nunca pude amar a nadie, hay diferentes islas, yo estoy en una y la isla del amor está allá lejos, yo no sé cómo llegar, además seguro esa isla debe ser sucia". Se trabaja con el usuario las distintas formas del amor, que quizá aún no había explorado el amor de pareja, pero si nos había narrado varias escenas donde había actuado desde un polo amoroso con familiares directos y amigos. De una forma sofisticada, el usuario manifiesta que no puede con esto solo, pide que se le acompañe, las islas son una porción de tierra rodeada de agua por todas partes, en este caso, nuestra tarea fue guiarle para que él descubra un puente para que las islas se conecten entre sí. Más adelante, él afirma: "estoy sintiendo cosas, tengo miedo", su relato nos permite abordar sus rasgos más obsesivos, su necesidad de tener un excesivo control, para él lo vinculado a la sexualidad es descontrolado e incierto, no le gusta la idea de lidiar con la incertidumbre, de saltar a una piscina sin saber si hay agua adentro, de poder resultar herido. Al poco tiempo, inicia una sesión manifestando: "tengo una revolución interna, me siento raro, estoy nervioso, creo que me gusta una persona, un varón, me atrae su personalidad, es muy diferente a mí". Y continúa con su relato: "Yo estaba en mi isla tranquilo y me empezaron a tirar cocos en la cabeza desde la isla de enfrente, no me gustó, los cocos vinieron de forma invasiva". Él toma contacto con que le atrae alguien, es algo nuevo, se siente vivo pero aterrado al mismo tiempo, su construcción identitaria como sujeto asexual empieza a estar en crisis, en la dimensión vincular de la sexualidad, los cocos generalmente llegan de forma invasiva, pero también hay que estar abierto, preparado para que eso acontezca, en fin, el puente se empezaba a edificar. Al mes el usuario ingresa al consultorio muy risueño, es posible notar un brillo inédito en sus ojos, toma asiento y afirma: "Quiero decirles que estoy de novio, es una relación a distancia, él vive en España, pero hablamos todo el día", la consulta avanza, Y baja la mirada y con notoria vergüenza plantea: "no sé si quiero hormonizarme, mis genitales me daban asco, pero ahora ya no tanto, desde que hablo con él me estoy masturbando y creo que me gusta, que siento cositas (risa tímida), creo que soy homosexual". Esta sesión introduce nuevos emergentes y nos conduce a definir nuevos objetivos terapéuticos. Por un lado, lo virtual podría ser entendido como un factor protector, la distancia como un ensayo más controlado para experimentar su sexualidad, lo cual no lo hace sentirse tan expuesto, dado que, la posibilidad de un encuentro en la realidad material concreta no era posible a causa de que se trataba de dos menores de edad y con limitaciones económicas. Sin embargo, esta experiencia virtual, le permite trabajar sobre su socialización sexual, la necesidad de ensayar y poner en práctica los guiones sexuales compartidos por la mayoría, algo que en su historia de vida había descuidado por autodefinirse como asexual, pero ahora desea ser parte, se siente por primera vez un sujeto sexuado, está aprendiendo a ser sexual. El hecho de explorar la dimensión vincular de la sexualidad, le lleva a hacerse preguntas sobre su propia transgeneridad, se permite abrir la puerta a las prácticas masturbatorias y siente placer sexual. De pequeño le decían que masturbarse estaba mal, que era pecado, por lo cual, se dedica un tiempo a desmitificar esto y se intenta que no sienta culpa por hacerlo. A su vez, el usuario comprueba que la orientación sexual es dinámica, desde su perspectiva abandona la categoría asexual, ya no cree que sea su orientación, la que mejor lo describe y la sustituye por la categoría homosexual, descubre que le atraen los varones. En los siguientes encuentros el trabajo se enfoca en el proceso de reafirmación de su identidad genérica y en la construcción de su nueva identidad sexual, una identidad gay.

\section{Algunas consideraciones generales sobre el concepto de sexualidad}

Como categoría conceptual, la palabra sexualidad es utilizada desde mediados del siglo XVIII. EI concepto de sexualidad está en constante construcción y transformación. Es objeto de estudio de diversas disciplinas, siendo un campo que se caracteriza por estar en disputa por la posición 
hegemónica. La sexualidad es un aspecto central de los seres humanos a lo largo del ciclo de vida y se encuentra en la base de los procesos de salud-enfermedad (Ramos, Forrisi y Gelpi 2015). Los sujetos nacen como seres sexuados, se tiene sexualidad desde el nacimiento hasta la muerte. A través del ciclo de vida, las personas exploran cómo vivenciarla, piensan en torno al ejercicio de su sexualidad, analizan qué sienten respecto a ella, se presentan dudas y variaciones dependiendo de las diferentes necesidades. A nivel interpersonal, puede ser entendida como el conjunto de maneras diversas en que las personas se relacionan como sujetos sexuados con otros seres también sexuados, en intercambios, acciones y prácticas cargadas de sentido (Marzano, 2015). En su repercusión concreta en la vida cotidiana se manifiesta a través del deseo, del placer, del erotismo, de la atracción, de la necesidad de gustar, de sentirse atractivo e identificarse con algo y también, en ocasiones, en el deseo de reproducirse. El concepto incluye la relación de las personas con su cuerpo y corporalidad (López, Forrisi y Gelpi, 2015). La sexualidad siempre está atravesada por una o varias de estas motivaciones, generalmente vinculadas a uno de los dos fines: el erótico placentero y/o el reproductivo. Por los motivos antes expuestos, conceptos como la orientación sexual, la identidad de género y las prácticas sexuales integran la noción de sexualidad (OMS, 2018).

Hay tres dimensiones fundamentales que aportan al análisis de la sexualidad: significaciones, discursos y prácticas. La tensión entre las dimensiones se evidencia cuando no existe una armonía entre lo que una persona desea hacer, hace y dice hacer. Eso que se puede leer a nivel individual, puede observarse a nivel social, cuando aquello que se dice sobre sexualidad no condice con los significados que se les otorga a las prácticas, generando, por ejemplo, regímenes de doble moral. La relación entre estas tres dimensiones desde una mirada interseccional, debe ser analizada como producto de relaciones de poder: de clase, género, raza/etnia, generación y otras variables (López Gómez et. al, 2015).

La sexualidad como concepto, categoría y práctica ha sido objeto de intervención a lo largo de la historia y ha variado el enfoque y abordaje de la misma por parte de las distintas instituciones que tienen la función de socializarnos (Foucault, 1998). De todos modos, aún es posible afirmar que sigue habiendo una pedagogía disciplinaria en materia de sexualidad. Desde la perspectiva Foucaultiana, disciplinar es más que controlar, "es un ejercicio de poder que tiene por objeto los cuerpos y por objetivo su normalización" (Junqueira, 2013, p. 490). El disciplinamiento no se produce necesariamente mediante la prohibición sino por la promoción de cómo se debe ser, eso es lo que Foucault denomina biopoder (Louro, 2000). Desde el surgimiento del concepto sexualidad hasta la actualidad, se pueden identificar cambios en el discurso científico, en el discurso moralreligioso y en el discurso familiar acerca de la sexualidad, así como también, se reconoce que ninguno de estos discursos es homogéneo, existen tensiones, conflictos, rupturas, en fin, diversidad. A su vez, lo antes descrito, se vincula directamente con las normas sexuales y de género vigentes en una cultura determinada, en una sociedad en particular, en un momento socio-histórico específico, en un contexto político concreto, todo lo cual, opera en la definición de los permisos y prohibiciones, de las habilitaciones o represiones, tanto a nivel de las representaciones como a nivel de los sentidos y significados que se adjudican a ciertos comportamientos sexuales (Weeks, 1988).

De hecho, aunque en la contemporaneidad, especialmente en occidente, la sexualidad haya dejado de ser exclusivamente un tema tabú, para convertirse en parte en un fetiche moderno, en un producto a ser consumido, en algo capturado por la lógica capitalista, que ha permitido resignificar lo que se entiende por público/privado o por intimidad, siguen activos así y todo diferentes mecanismos cotidianos de vigilancia, regulación, control, intervención y castigo que tienen como 
objetivo la normalización de la diferencia o al menos de algunas de ellas (Gelpi, Pascoll y Egorov, 2019; Han, 2014; Giddens, 1992). Estos mecanismos con sus variaciones de frecuencia e intensidad acaban impactando de distinta manera en la biografía de los sujetos. En este contexto, ciertas disciplinas científicas, por ejemplo, la medicina, la psicología y la psiquiatría, siguen teniendo una función social específica en cuanto a la tarea de evaluar, diagnosticar y a veces tratar ciertos comportamientos, cuerpos e identidades, incluso en ocasiones vulnerando los derechos humanos de las personas. Es en este contexto que la asexualidad muchas veces es interpretada como un enigma, como una extrañeza, algo que escapa al entendimiento, que desafía a las teorías y a los paradigmas en este campo, algo que no es esperado ni deseado. Mientras en occidente en las últimas décadas, se produjeron avances legales y socioculturales que permitieron la despatologización de ciertas identidades sexuales, el reconocimiento de los derechos de las identidades trans y un aumento en la aceptación social de la homosexualidad (Barrientos, 2016), todo lo que rodea a la asexualidad se caracteriza por el desconocimiento. Generalmente el discurso científico, el moral-religioso y el familiar sobre sexualidad suelen coincidir en concebir a la asexualidad como una expresión patológica de la sexualidad y se termina buscando ayuda profesional para revertirla. Esta realidad es curiosa y deja a los sujetos que se autoidentifican como asexuales en una especial situación de desventaja porque mientras el discurso científico hegemónico, el discurso familiar hegemónico y algunos representantes del discurso moral-religioso aceptan y legitiman cada vez en mayor medida por ejemplo a la homosexualidad como una orientación sexual válida, en cuanto al entendimiento de la asexualidad, sobran las interrogantes, las dudas y el rechazo. Este estado de situación, nos permite comprender que, por un lado, la noción de libertad sexual sigue siendo restrictiva y se caracteriza por su fuerte componente ilusorio aún en democracia, por otro lado, nos acerca a la idea de que la propia diversidad sexo-genérica ha sido normalizada y el proceso de normalización ha sido excluyente de algunos sujetos, cuerpos e identidades.

\section{Aportes para aproximarnos al concepto de asexualidad}

Los primeros antecedentes sobre estudios que abordan la temática se ubican en los años 70' del siglo pasado. En este sentido, la pionera fue Myra Johnson, con su trabajo titulado "Asexual and Autoerotic Women: Two invisible groups" publicado en 1977. A pesar de que su muestra estuvo conformada exclusivamente por mujeres, sus aportes fueron valiosos y concluye que existe un grupo de personas que independientemente de factores como sus condiciones físicas, psicológicas, ideológicas, religiosas o historia sexual, no presentan interés en involucrarse en prácticas sexuales vinculares. Recién en el año 2001 se crea el sitio web AVEN (the asexual visibility and education network). Este sitio dedicado al tema permitió que el conjunto de personas autoidentificadas como asexuales lograra adquirir mayor visibilidad social a escala global, principalmente dentro de la comunidad LGBT+.

La asexualidad es un objeto de estudio reciente. Históricamente los términos utilizados para describir a estas personas se relacionan con categorías diagnósticas de tipo psiquiátrico, como las que son mencionadas en el DSM V (APA, 2013), por ejemplo, el trastorno de deseo sexual hipoactivo en varones o el trastorno de interés/excitación en mujeres (Brotto y Yule, 2016). Desde este enfoque la asexualidad no se considera una orientación sexual o una identidad sexual, sino más bien un trastorno. Sin embargo, no hay unanimidad al respecto, hay científicos que la pretenden legitimar y despatologizar. En base a este escenario, se puede afirmar que, la asexualidad, tiene un rasgo disruptivo, abre un debate académico pertinente, coloca en el foco conceptos muy naturalizados 
como el deseo sexual, la atracción sexual, las prácticas sexuales y la sexualidad en general como conducta basal en los seres humanos.

Álvarez (2010) define a la asexualidad como aquello donde no existe ni impulso ni respuesta sexual. Un modo de ser cuyo rasgo más significativo es la falta o el nulo deseo sexual. Conley-Fonda y Leisher (2018) de acuerdo a una revisión bibliográfica sobre el tema, plantean que la asexualidad, se puede definir como la falta o carencia de deseo o atracción sexual, es decir las personas asexuales nunca experimentaron este sentimiento. También van a sostener que hay diferentes rangos o modos de vivenciar la asexualidad, lo cual no implica necesariamente abstenerse completamente de tener prácticas sexuales (muchas personas asexuales mantienen prácticas con sus parejas), sino que el centro está en cómo cada sujeto experimenta y significa sus propias prácticas. Por otra parte, la AVEN (Asexual Visibility and Education Network) propone una definición de asexualidad que se caracteriza por la diversidad de vivencias dentro de las personas autoidentificadas como asexuales, las cuales no la entienden como un problema, sino como un punto de partida distinto para enfrentar ciertas situaciones en comparación con las personas que si experimentan deseo sexual (AVEN, s/f). El espíritu de esta definición coincide con la de Scherrer (2008), quien, a partir de un estudio de casos, concluye que, cada participante, tiene una definición distinta de lo que considera asexual y el hecho de autoidentificarse como asexual no significa que no materialicen prácticas sexuales genitales o que no tengan actividades que involucren otras zonas erógenas. En las últimas dos definiciones se visualiza un enfoque, una perspectiva, que pretende rescatar los elementos positivos relativos al acto de definir la asexualidad a diferencia del resto de las definiciones, donde se parte del supuesto de considerar a la asexualidad como la falta o carencia de algo, dejando en cierta forma de lado las distintas variables que interactúan en cualquier comportamiento de índole sexual (Van Houdenhove, Enzlin y Gijs, 2017). Igualmente varios autores y autoras concuerdan con Álvarez (2010), con relación a que el grupo de personas asexuales, es un grupo minoritario de la sociedad, cuyo rasgo distintivo hace que entiendan y experimenten su sexualidad de un modo diferente al de la mayoría de las personas, por lo cual, a veces su realidad es poco compartible y comprensible para los demás. Asimismo, la misma se presenta tanto en personas cisgénero como transgénero, tanto en varones como en mujeres, aunque probablemente el juicio de valor que realiza la sociedad varíe dependiendo el género de la persona, especialmente si se consideran las expectativas que se depositan en varones y en mujeres en tanto seres sexuados, por ejemplo, los varones suelen ser reconocidos cuanta más actividad sexual informen, ya que, cumplir con el modelo hegemónico de sexualidad es visto como sinónimo de virilidad (Connell, 2003). Otra variable que condiciona es la generación, habitualmente se suele naturalizar, normalizar y fomentar el ejercicio activo de la sexualidad especialmente durante la adolescencia y juventud.

De todos modos, como habrán notado, definir a la asexualidad no es una tarea sencilla, es deseable adoptar una posición crítico-reflexiva, revisar nuestras propias creencias al respecto, nuestros marcos cognitivos e interpretativos a la hora de intervenir y de producir conocimiento en materia de sexualidad, disponer de energía psíquica para problematizar premisas construidas como verdades absolutas que ya no suelen ser cuestionadas dentro de la cultura científica y de las disciplinas y subdisciplinas que involucran al estudio de la sexualidad humana, así como también, se trata de aceptar la heterogeneidad de posturas epistemológicas y políticas de quienes construyen a la asexualidad como objeto de estudio, teniendo la flexibilidad de reconocer que dados los cambios permanentes que se producen en la trama social, cualquier conclusión sobre este fenómeno será parcial. Por los motivos antes expuestos, es válido preguntarse: ¿la asexualidad es una orientación sexual al igual que lo es la heterosexualidad o la homosexualidad? ¿es una identidad sexual como es la identidad gay o lesbiana? ¿es un trastorno sexual en base a una falla en el desarrollo psico- 
sexual y/o a la exposición a un evento traumático? ¿es posible ser asexual? ¿'se es asexual o se está asexual? ¿la asexualidad puede ser una condición permanente durante todo el ciclo de vida o más bien una fase momentánea?

A su vez, es fundamental mantener una vigilancia epistémica que ayude a evitar cometer violencia epistémica ${ }^{4}$ hacia las personas que se autoidentifican como asexuales. Quienes escribimos este artículo no nos identificamos como asexuales, no somos parte de dicha comunidad y sus integrantes tampoco son los principales productores de conocimiento científico sobre el tema, todos estos pueden ser entendidos como factores de riesgo para la comunidad asexual, por esta razón, se aclara que no es nuestro objetivo violentar la subjetividad ni las vivencias de este conjunto de personas, sino por el contrario, visibilizar sus identidades, sus conflictos, sus declaraciones de existencia (Pérez, 2019; Radi, 2019).

A continuación, se presentan argumentos que apoyan y rechazan la idea de entender a la asexualidad como una orientación sexual, una identidad sexual o un trastorno sexual. El objetivo de este bloque es abrir la discusión, ofrecer herramientas para que los lectores puedan reflexionar, facilitar el proceso de elaboración de nuevas interrogantes, ensayar algunas respuestas posibles y dejar en evidencia las ventajas y limitaciones de cada uno de estos enfoques teóricos.

\subsection{La asexualidad entendida como una orientación sexual}

Una definición clásica de orientación sexual sería aquella que refiere al sexo hacia donde una persona orienta su deseo erótico-afectivo-sexual. La orientación sexual de los sujetos es dinámica, no es fija y las prácticas sexuales que tienen las personas no definen su orientación. Esta no es una opción, no se elige, depende de factores que escapan a la consciencia. La clasificación tradicional más usada, es la que considera tres orientaciones: la heterosexualidad, la bisexualidad y la homosexualidad. Sin embargo, dicha clasificación está sujeta a una revisión constante, dado que, en el último tiempo, han surgido nuevas categorías que dan cuenta de la complejidad de la sexualidad humana, por ejemplo, las más resonadas son la pansexualidad y la asexualidad (Ramos, Forrisi y Gelpi, 2015).

Para un conjunto de expertos, la asexualidad sería una "nueva" orientación sexual, para otro grupo de académicos aún no alcanza ese estatus. A su vez, esta discusión tampoco está resuelta dentro de los propios movimientos sociales. Si se analiza la asexualidad desde los lentes del concepto de orientación sexual, en primer lugar, se podría decir que, ambas categorías están en conflicto porque la orientación se sustenta en la idea natural de atracción o deseo sexual como base etimológica fundante. En cambio, en el caso de la asexualidad, dicha atracción o deseo no estaría presente. Por otra parte, si se toma en consideración que la orientación no es una elección, la asexualidad entra dentro de su paraguas conceptual. En esta línea, no se debe confundir la asexualidad con la castidad. La castidad es una elección, la asexualidad no. Hay personas que manifiestan un conflicto a causa de su asexualidad y otras la normalizan y la vivencian con tranquilidad. Los conflictos psíquicos pueden tener un origen interno o externo, por ejemplo, en ocasiones son las propias personas asexuales quienes creen que serlo no es algo "normal", mientras que, en otros casos, es el entorno cercano a la persona que coopera para que se produzca la emergencia de un conflicto ante el señalamiento de que esto llamado asexualidad es un problema y por ende se debe buscar una solución de la mano de ayuda profesional. En ambos casos, este "conflicto", se puede comparar con

\footnotetext{
4 "Refiere a las distintas maneras en que la violencia es ejercida en relación con la producción, circulación y reconocimiento del conocimiento: la negación de la agencia epistémica de ciertos sujetos, la explotación no reconocida de sus recursos epistémicos, su objetificación, entre muchas otras cosas" (Perez, 2019, p.82).
} 
otros procesos inherentes al descubrimiento de una orientación no heterosexual y la tarea de construir una identidad disidente, donde en un principio existe un sentimiento relacionado con el hecho de sentirse diferente, muchas veces propiciado por un entorno que arrastra a la confusión y que lleva a la persona a necesitar respuestas (Van Houdenhove, Enzlin y Gijs, 2017; Brotto y Yule, 2016). Por otro lado, si la discusión se centra en el potencial dinamismo inherente a la orientación sexual, se puede reflexionar acerca de ciertas expresiones pasajeras de asexualidad, por ejemplo, ante una ruptura amorosa, ante un duelo que se está elaborando, ante una cotidianeidad atravesada por altos niveles de estrés, entre otras posibilidades. Ante este escenario, no se estaría hablando de una condición asexual permanente, sino de una condición asexual contextual.

El concepto de orientación sexual en general es amplio en cuanto a su alcance, dentro de él se contemplan aquellas situaciones donde se produce una atracción predominantemente afectiva, amorosa, romántica, sin necesidad de materializar prácticas sexuales hegemónicas. En este sentido, en varios estudios, se insiste en que, las personas asexuales hacen uso de distintas formas de relacionamiento con otras personas e incluso con sus parejas, no son un grupo homogéneo. Mientras para algunas personas asexuales el simple hecho de darse la mano o abrazarse ya les genera incomodidad y es una idea inadmisible, otras disfrutan estos actos, pero siguen sin considerarlos sexuales (Scherrer, 2008). Algunas preguntas que se configuran como válidas son: ¿qué entienden por sexualidad las personas que se autoidentifican como asexuales? ¿qué significa para ellas ser seres sexuados? ¿cómo experimentan su asexualidad? ¿y cómo la expresan?

Al retomar nuevamente el estudio de Scherrer (2008), nos encontramos con que muchas personas asexuales se definen como heterosexuales, homosexuales o bisexuales (aunque sin ir contra los testimonios de estas personas, habría que aclarar que se trata de una orientación afectiva y no sexual). Dicho de otro modo, el estudio, nos permite aproximarnos a conocer los modos de relacionamiento que suelen tener las personas asexuales y nos acercan a la idea de que autoidentificarse como asexual no impide estar en pareja ni en contacto emocional con otras personas, simplemente, desde el punto de vista de los participantes de dicho estudio, no existe deseo de un acto sexual hegemónico, lo cual es considerado como algo más que sucede en la pareja y no como una cuestión central. Este último punto lo aborda uno de los máximos exponentes de la temática, Anthony Bogaert, él plantea una distinción entre orientación sexual y orientación afectiva, asegurando que las personas asexuales no necesariamente sienten rechazo a un vínculo emocional (hay casos que sí). Sus aportes nos conducen a creer que algunas personas tienen la capacidad de disociar la atracción emocional/afectiva/amorosa/romántica de la sexual, todo lo cual, complejiza aún más el concepto de orientación sexual (Bogaert, 2012). Además, se debe recordar que, teóricamente nuestras prácticas sexuales no definen nuestra orientación sexual.

\subsection{La asexualidad entendida como una identidad sexual}

Existen diferentes teorías sobre el concepto de identidad sexual. En este artículo se adhiere a una definición concebida dentro del campo de los gay studies. En esta línea, se asocia el concepto a un componente político, donde los sujetos se caracterizan por politizar su propia sexualidad con el objetivo de hacer visible en espacios públicos ciertas identidades históricamente oprimidas, por ejemplo, la identidad gay o lesbiana. Esta perspectiva permite abrir un fuerte debate sobre si la heterosexualidad es una identidad sexual o no (Savin-Williams, 2009). De hecho el fenómeno de construir una identidad en función de los comportamientos afectivo-sexuales es algo reciente, que data de las primeras décadas del siglo XX (Guasch, 2007). Sin embargo, en la contemporaneidad, especialmente en las generaciones más jóvenes y en ciertos contextos específicos, se comienza a notar una tendencia de criticar la necesidad de colocar etiquetas sexuales. En ocasiones la acción 
de colocarse una etiqueta surge como una necesidad de la propia persona ante la incertidumbre de no poder responder algunas preguntas existenciales sobre su sexualidad, pero a veces las personas no tienen interés en autoetiquetarse, pero sienten la presión del entorno, un entorno que exige respuestas, verdades. En materia de diversidad sexo-genérica, existen teorías clásicas como la de Richard Troiden o Vivienne Cass sobre el proceso de construir o reforzar la identidad sexual. Todas ellas coinciden en que habitualmente la construcción de la identidad sexual suele darse durante la adolescencia. Gil Herdt y Andy Boxer (Citados en Savin-Williams, 2009) señalan que la cuestión de construir una identidad sexual tiene rasgos de un autodiálogo, un proceso interior reflexivo por el que las personas reconocen y luego aceptan sus deseos. Se presenta una evolución, donde una persona pasa del "quizá sea" al "probablemente sea" luego a "sin duda soy" y finalmente, si es un desarrollo saludable, el proceso se suele cerrar con un "orgullosamente soy". En el caso de las personas que se autoidentifican como asexuales este proceso se torna más dificultoso que para otras identidades porque actualmente existen pocos modelos disponibles de asexualidad, la comunidad asexual está algo aislada o fragmentada dentro del movimiento social y porque a nivel social persiste un profundo desconocimiento sobre el tema que favorece la emergencia de preconceptos negativos sobre la asexualidad. En una sociedad que mercantiliza la sexualidad, la asexualidad es una de las expresiones periféricas menos deseada en nuestra cultura. Asimismo, hay personas que se autoidentifican como asexuales, pero tienen actividad sexual, por lo cual, sus prácticas no están en sintonía con la categoría que creen se ajusta mejor a ellos. Igualmente, las categorías de identidad sexual que adoptan las personas reflejan los valores de la relación, la pertenencia, la diferencia y la diversidad. Ofrecen continuas posibilidades para la invención y la reinvención, son procesos abiertos por los que se puede producir el cambio (Weeks, 1995). Además, siempre se debe respetar la categoría identitaria que escoge cada persona para narrarse al mundo exterior porque de lo contrario se podría estar violentando a esa otredad.

Al retomar la dimensión de la politización de las identidades, es válido preguntarse: ¿cuáles son las demandas y necesidades del colectivo de personas asexuales? ¿qué denuncias sociales han realizado como grupo organizado? ¿qué causas defienden? ¿qué motivos les unen? ¿qué derechos creen que les vulneran o les han vulnerado? ¿por qué luchan? ¿qué función cumplen dentro del movimiento LGBT+? ¿quiénes son sus aliados estratégicos? ¿quiénes son sus detractores en la arena pública? ¿los decisores de políticas de género y diversidad reconocen a las personas asexuales y a la asexualidad en general como objeto de políticas públicas? ¿en qué contexto? (López y Platero, 2019).

\subsection{La asexualidad entendida como un trastorno sexual}

A lo largo de la historia disciplinas científicas como la psiquiatría, la psicología y la sexología, han intervenido sobre la sexualidad humana y han creado un conjunto de teorías, técnicas y métodos de trabajo en esta materia. Generalmente este conjunto de disciplinas ha tenido el rol de dictaminar qué manifestaciones y expresiones de la sexualidad son consideradas sanas y cuáles son patológicas. Los criterios de evaluación, diagnóstico y tratamiento han ido variando con el tiempo, por ejemplo, hasta el 1973 la homosexualidad fue considerada una enfermedad mental.

Freud (1905) plantea que existe un desarrollo psicosexual esperable en los sujetos. Al culminar la latencia, se entraría en un momento del ciclo de vida entre la pubertad y la adolescencia, que se comienza a caracterizar por el ejercicio de una sexualidad genital. Este novedoso modo de descubrir una sexualidad más propia del mundo adulto pasa a tener un rol central en la biografía de las personas, en esta etapa, se suele descubrir la orientación sexual, se reafirma la identidad sexual y también la identidad de género. Es esperable que se produzcan las primeras prácticas 
masturbatorias, que se constate un mayor consumo de contenido pornográfico y que se concrete la tan ansiada iniciación sexual.

Desde este punto de vista, la asexualidad solo tendría lugar en tanto una desviación de lo esperado, de lo sano. Por este motivo, Conley-Fonda y Leisher (2018) hablan de las consecuencias negativas que recaen sobre las personas autoidentificadas como asexuales cuando estas informan su realidad. Un grupo nada despreciable de personas que forman parte del entorno cercano, así como profesionales de la salud, consideran a la asexualidad solamente como un trastorno o una disfunción. El concepto disfunción sexual hace referencia a la alteración de una o varias de las fases de la respuesta sexual, que de forma persistente podría causar problemas o molestias en los sujetos (Sánchez et. al, 2007).

Actualmente, el DSM V (APA, 2013) describe la existencia de un trastorno de deseo sexual hipoactivo en varones o trastorno de interés/excitación en mujeres, en donde el punto de encuentro entre ambos trastornos, salvando algunas diferencias, parte de la base de una disminución o inexistencia tanto del interés y excitación sexual como del inicio de actividades sexuales. En este sentido, ambos trastornos parten de un supuesto hegemónico de normalidad o basalidad del deseo sexual. Desde esta perspectiva teórica, la asexualidad o al menos su característica principal que es la inexistencia de deseo sexual es vista como patológica, algo que impacta negativamente en la salud sexual de las personas y para afirmarlo se apoyan por ejemplo en más de 100 años de producción sobre psicología del desarrollo.

Sin embargo, se debe aclarar que, el DSM $V$ también expresa que, en caso de que las personas manifiesten una identidad asexual, el diagnostico deja de aplicarse (APA, 2013). Este abordaje apoya la idea de que la asexualidad es una identidad sexual sana y posible, siempre y cuando la inexistencia de deseo sexual no le ocasione malestar o conflicto psíquico a las personas. Igualmente, si se considera lo expuesto en este apartado, se hace evidente que en este punto se expresan tensiones dentro del propio manual.

Todo lo antes abordado nos conduce a la discusión sobre la autodeterminación personal de quienes se identifican como asexuales, el foco aquí es qué sucede con el deseo y el derecho a ser y hacer de este conjunto de personas, aunque esto también tiene un límite epistémico, político y ético porque no se pueden legitimar todas las causas en la esfera de la sexualidad humana. Pero a pesar de la lucha por el reconocimiento de la asexualidad, claramente aún hay un núcleo duro que no está dispuesto a debatir sobre algo que pareciera incuestionable, que naturalmente las personas tienen deseo sexual. Esto podría abrir una discusión muy potente entre los representantes de los paradigmas dominantes sobre sexualidad, por un lado, el esencialista, que no es lo mismo que el determinismo biológico y por el otro lado el construccionismo. Incluso creemos que es viable interrogarse si desde un paradigma dominante todas las expresiones y manifestaciones de la asexualidad podrían ser entendidas como patológicas o si habría excepciones. Además, se cree que es válido preguntarse: ¿por qué hay profesionales de la salud que se rehúsan a reconocer a la asexualidad como una forma más de vivenciar y ejercer la sexualidad? ¿qué elementos consideran para argumentar su postura?

Asimismo, es importante saber diferenciar las prácticas vinculadas al autoerotismo de las prácticas vinculares, es decir, las que involucran a terceros. En esta línea, en algunos estudios, los participantes informaron que se masturban, aunque muchos de ellos no consideran a la masturbación como una actividad sexual, simplemente la utilizan como una práctica relajante o lo 
hacen porque sienten ganas, pero sin asociar la acción a ningún otro estímulo. Esta realidad de algunas personas asexuales nos muestra la enorme diversidad con relación a lo que llamamos asexualidad, así como también, se evidencian las diferencias en cuanto a cómo significan las experiencias sexuales las personas, ya que, mientras para la mayoría de las personas, algunas prácticas son significadas como sexuales, para las personas asexuales estas no guardan ninguna relación con la sexualidad. Este tipo de disyuntiva también se suele presentar dentro de la comunidad LGBT+ a la hora de informar la iniciación sexual, por ejemplo, el sexo oral no se suele considerar como la iniciación porque aún el rasgo coitocéntrico y falocéntrico sigue teniendo un fuerte peso (Savin-Williams, 2009).

Por último, como se ha mencionado anteriormente, para muchas personas asexuales no hay nada de problemático en serlo, si no hay angustia, si no hay malestar psíquico, si no hay conflicto, los profesionales de la salud tienen poco margen para intervenir, luego están los casos donde las personas presentan lo anterior pero por los señalamientos y los juicios de los otros, allí la persona llega a un espacio de consulta por recomendación de terceros, pero no necesariamente hace una construcción de demanda, por lo cual, la adherencia al espacio es baja. Luego están las personas que se autoidentifican como asexuales pero no están conformes con esto, buscan ayuda profesional por motivación propia y anhelan un cambio en cuanto a sus comportamientos sexuales. En esta última escena se abre la puerta para que en estos casos psicólogos, sexólogos y/o psiquiatras tengan mayor libertad para intervenir, la pregunta es: ¿desde qué paradigma? ¿qué métodos y técnicas se usan habitualmente? ¿cómo influye la ética profesional en todo esto? ¿en qué medida dichas intervenciones benefician a las personas asexuales?

\section{El proceso de identificación con la categoría asexual en el marco de una historia de abuso sexual infantil}

Ambos usuarios que fueron atendidos en CRAm y se autoidentifican como asexuales comparten un evento traumático en sus historias de vida, haber sido víctimas de abuso sexual infantil (ASI). Según Berlinerblau (2016) el ASI implica toda interacción sexual en la que el consentimiento no existe o no puede ser dado, independientemente de si el niño, niña o adolescente entiende la naturaleza sexual de la actividad e incluso cuando no muestra signos de rechazo. EI ASI se caracteriza por prácticas sexuales violentas, que contemplan manoseos, exposición de los órganos sexuales, masturbacion frente a un niño, niña o adolescente y violacion ${ }^{5}$, entre otras. Estas prácticas son impuestas por una persona adulta, en la mayoría de los casos cercanas a las víctimas. Generalmente se utilizan amenazas, manipulaciones, se instalan pactos de secreto y silencio, se produce violencia psicológica. El ejercicio de la fuerza puede estar o no, pero lo que si siempre se presenta es un ejercicio de asimetría de poder (Ramos, Finzi y Gualdoni, 2018).

A nivel general, el ASI, se considera un tipo específico de violencia sexual. La Organización Mundial de la Salud (OMS) define a la violencia sexual como "todo acto sexual, la tentativa de consumar un acto sexual, los comentarios o insinuaciones sexuales no deseados, o las acciones para comercializar o utilizar de cualquier otro modo la sexualidad de una persona mediante coacción por otra persona,

\footnotetext{
${ }^{5}$ La violación sexual se define como la penetración vaginal, anal u oral con el pene o cualquier otro tipo de objeto, en forma abrupta, con violencia física, violencia emocional y control de la situación mediante el uso de la fuerza. Este tipo de abuso se identifica por la presencia de lesiones en el área genital, anal o en otras regiones del cuerpo y por acompañarse de manifestaciones emocionales de ansiedad o estrés agudo" (García et. al, 2013, p. 289).
} 
independientemente de la relación de ésta con la víctima, en cualquier ámbito, incluidos el hogar y el lugar de trabajo" (OMS, 2013, p. 2). Este tipo de violencia también puede ocurrir cuando la persona no se encuentra en condiciones de dar su consentimiento (por ejemplo bajos los efectos del alcohol o algún otro tipo de sustancia psicoactiva) (UIG, 2017). En la actualidad también hay violencias sexuales que se producen en entornos digitales, por ejemplo, la acción de enviar "nudes" o "packs" a otra persona sin su consentimiento o realizar una práctica de sextorsion o sexrevange. A su vez, la violencia sexual es un tipo específico de violencia basada en género, es un grave problema de derechos humanos y de salud pública, el cual afecta a un número importante de personas alrededor del mundo. Aunque cualquier persona puede ser afectada por este tipo de violencia, las poblaciones más afectadas suelen ser las niñas, adolescentes y mujeres adultas. En la mayoría de los casos, los que ejercen este tipo de violencia, son varones conocidos por las víctimas (familiares directos, personas convivientes, pareja, compañeros de trabajo o escuela, amigos, etc.) (García y Cerda, 2010).

La violencia sexual suele guardar una relación directa con ciertos mecanismos patriarcales de control, subordinación y reafirmación de la dominación masculina. Las mujeres que informan ausencia de deseo sexual o que tienen un deseo no heterosexual, ajeno a la mirada masculina, suelen estar más expuestas a la violencia sexual. Muchas mujeres lesbianas, bisexuales, asexuales y personas trans en general, son castigadas socialmente por desviarse de la "norma", acaban sufriendo en sus cuerpos virulentos dispositivos de castigo y corrección, que van desde la violencia simbólica ("a ti lo que te hace falta es una buena...") a una violencia atroz en el marco de una estructura profundamente heteronormativa, cisnormativa y homo-lesbo-transfóbica, por ejemplo, prácticas que ponen en riesgo la integralidad de las personas, como son las violaciones correctivas (De la Hermosa y Usaola, 2018).

Asimismo, es fundamental comprender el recorrido histórico que se hizo para arribar a este concepto relativamente moderno. Antes los niños, niñas y adolescentes estaban involucrados en las actividades sexuales de los adultos y esto no era entendido como un problema. La sensibilidad moderna, la creación de nuevas etapas del ciclo de vida con características propias y los aportes de algunas disciplinas científicas, permitieron empezar a pensar en las buenas prácticas de crianza, qué prácticas son tolerables y cuáles no, cuáles son deseadas y cuáles se deben evitar, en este contexto, el ASI es un concepto que paulatinamente comenzó aparecer ya no únicamente en el campo de la salud sino también en el del derecho. Dicho de otro modo, se pasó de la naturalización y normalización al cuestionamiento y sanción moral/legal. En esta dirección, empezó a existir campo fértil para reconocer a niños, niñas y adolescentes como sujetos de derecho ${ }^{6}$ (Balta y Moreno, 2015).

A pesar de que hay una mayor sensibilidad sobre el problema, un consenso social sobre que este tipo de prácticas son totalmente nocivas para la salud de niños, niñas y adolescentes, su prevalencia a nivel nacional sigue siendo importante. Según Lozano (2017) en el 2016 la Administración de los Servicios de Salud del Estado (ASSE) contabilizo que el $20 \%$ de los casos de violencia sobre niños, niñas y adolescentes que ingresaron al Instituto del Niño y Adolescente del Uruguay (INAU) fueron por violencia sexual y el $74 \%$ de ingresos por esta causa son de niñas. En el mismo estudio, entre el

\footnotetext{
6 Antes eran muy frecuente instrumentos de castigo, que aún hoy pueden encontrarse y en algunos casos se siguen utilizando, por ejemplo: látigos, palas, bastones o varas de hierro, y la amenaza con figuras monstruosas. "El infanticidio y el abandono de niños eran prácticas absolutamente toleradas, y en las que ya se podían distinguir discriminaciones basadas en el género (si se daba a luz a un hijo varón, se lo conservaba, pero a una hija mujer se la abandonaba) y las diferencias (se abandonaba a los hijos con alguna situación de discapacidad)" (Balta y Moreno, 2015, p. 12).
} 
$3 \%$ y el $24 \%$ de las mujeres participantes, relató que su primer encuentro sexual fue forzado, encuentros que en su mayoría acontecieron en la adolescencia. A su vez, aunque no hay estadísticas oficiales, se tiene conocimiento que las personas $L G B T+$ son un grupo de riesgo para sufrir este tipo de violencia específica (CIDH, 2015). La Comisión Interamericana de Derechos Humanos en su informe "Violencia contra Personas Lesbianas, Gay, Bisexuales, Trans e Intersex en América" es concluyente con relación a la prevalencia de violencia sexual dirigida hacia la población LGBT+, principalmente dichas acciones son cometidas con el fin de: a) "castigar" la diferencia; b) corregir desde la perspectiva de los opresores el atropello a las normas de género y sexuales que realizan las personas de la comunidad LGBT+; c) proteger el sistema binario que la sociedad impone en cuanto al género; d) asegurar que cada quien ejerza el rol de género asignado a nivel social.

Todas las expresiones y manifestaciones de la violencia sexual, sean episodios únicos o sostenidos en el tiempo, producen efectos bio-psico-sociales negativos en las víctimas. Un factor que se debe considerar es el momento del ciclo de vida en el que se produce la experiencia de abuso. Los riesgos para la salud integral de las personas suelen incrementarse especialmente cuando dichas experiencias acontecen a edades tempranas porque el aparato psíquico se ve superado por estas situaciones del orden de lo traumático que acaban afectando el desarrollo psicosexual y se producen secuelas a largo plazo. En cuanto a estas, se puede afirmar que no suelen abarcar necesariamente a la mayoría de víctimas de ASI. Para el desarrollo de las mismas van a incidir otra serie de factores tales como vivenciar otras situaciones traumáticas, la duración del abuso, la cercanía de la víctima con su agresor (el impacto traumatogénico que puede aparecer en las víctimas suele ser mayor ante abusos intrafamiliares. Se generan sentimientos dispares y contradictorios con respecto a la confianza, la protección, el apego que se espera y se vivencia en relación a vínculos tan cercanos y fundantes como los familiares), la repercusión que tuviera la develación del abuso en el entorno cercano, entre otros. Algunas de las secuelas a largo plazo son: síntomas de depresión y/o ansiedad, estrés postraumático, control inadecuado de la ira, intentos de autoeliminación, consumo problemático de sustancias, disfunciones sexuales e incapacidad de disfrute (Villanueva, 2013; Echeburúa y De Corral, 2006).

Es clave entender que involucrar a niños o niñas emocionalmente inmaduros, dependientes, incapacitados para dar su consentimiento, en actividades sexuales propias del mundo adulto, que violan los tabúes de los roles familiares y de la cultura a la que se pertenece, puede propiciar una desestructuración psíquica importante (Quenan y Samudio, 2013). De hecho, en muchos casos, las situaciones de abuso no salen a la luz porque las víctimas enuncien y denuncien lo sucedido, sino que se descubre en base a la presencia y detección de indicadores físicos, psicológicos, comportamentales y sexuales asociados al ASI. Echeburúa y De Corral (2006) elaboraron un listado que se comparte a continuación en la siguiente figura: 


\begin{tabular}{|c|c|c|}
\hline \begin{tabular}{|l} 
a. Indicadores físicos \\
\\
- Dolor, golpes, \\
quemaduras o heridas \\
en la zona genital o \\
anal. \\
- Cérvix o vulva \\
hinchadas o rojas. \\
- Semen en la boca, en \\
los genitales o en la \\
ropa. \\
- Ropa interior rasgada, \\
manchada y \\
ensangrentada. \\
- Enfermedades de \\
transmisión sexual en \\
genitales, ano, boca u \\
ojos. \\
- Dificultad para andar y \\
sentarse. \\
- Enuresis o encopresis.
\end{tabular} & $\begin{array}{l}\text { b. Indicadores } \\
\text { comportamentales } \\
\text { - Pérdida de apetito. } \\
\text { - Llantos frecuentes, sobre } \\
\text { todo en referencia a } \\
\text { situaciones afectivas o } \\
\text { eróticas. } \\
\text { - Miedo a estar sola, a los } \\
\text { hombres o a un } \\
\text { determinado miembro de la } \\
\text { familia. } \\
\text { - Rechazo al padre o a la } \\
\text { madre de forma repentina. } \\
\text { - Cambios bruscos de } \\
\text { conducta. } \\
\text { - Resistencia a desnudarse y } \\
\text { bañarse. } \\
\text { - Aislamiento y rechazo de las } \\
\text { relaciones sociales. } \\
\text { - Problemas escolares o } \\
\text { rechazo a la escuela. } \\
\text { - Fantasias o conductas } \\
\text { regresivas (chuparse el } \\
\text { dedo, orinarse en la cama). } \\
\text { - Tendencia al secretismo. } \\
\text { - Agresividad, fugas o } \\
\text { acciones delictivas. } \\
\text { - Autolesiones o intentos de } \\
\text { suicidio. }\end{array}$ & $\begin{array}{l}\text { c. Indicadores en la } \\
\text { esfera sexual } \\
\text { - Rechazo de las caricias, } \\
\text { de los besos y del } \\
\text { contacto físico. } \\
\text { - Conductas seductoras, } \\
\text { especialmente en niñas } \\
\text { - Conductas precoces o } \\
\text { conocimientos sexuales } \\
\text { inadecuados para su } \\
\text { edad. } \\
\text { - Interés exagerado por } \\
\text { los comportamientos } \\
\text { sexuales de los adultos. } \\
\text { - Agresión sexual de un } \\
\text { menor hacia otros } \\
\text { menores. } \\
\text { - Confusión sobre la } \\
\text { orientación sexual. }\end{array}$ \\
\hline
\end{tabular}

Fuente: Echeburúa y De Corral (2006)

Los dos usuarios que recibieron un acompañamiento y tratamiento psicológico desde el CRAm que se presentaron en otro pasaje del artículo tienen puntos en común: en ambas situaciones la experiencia de abuso aconteció durante la infancia, se trabajó sobre las secuelas a largo plazo, las cuales estaban activas aún en la actualidad, se caracterizaban por ser personas con dificultades para socializar, sentían culpa y vergüenza por lo sucedido, sentían rechazo por su propio cuerpo y por sus genitales (en el caso del adolescente trans este sentimiento es esperable porque a veces es algo característico en dichas identidades), tenían la autoestima disminuida, informaban distintos tipos de psicosomatizaciones, la sexualidad estaba significada como algo asqueroso, como algo que duele, que genera daño, se autoidentificaban como asexuales y mostraban conflictos con la dimensión vincular de la sexualidad. En esta línea, Franco et. al, (2013) sostienen que sufrir ASI puede impactar sobre la libido adolescente y sobre el desarrollo de una sexualidad genital. Estos autores parten del supuesto de que la relación de los adolescentes con su cuerpo y sexualidad es un proceso que viene desde la infancia, que adquiere otro carácter luego de la pubertad, pero que está marcado por todos los procesos que van constituyendo al sujeto como tal. Por este motivo, algunas víctimas de ASI, frecuentemente tienen el desafío de resignificar el evento como traumático luego de la pubertad, lo cual puede despertar en los sujetos una serie de recuerdos displacenteros que producen angustia. A partir de esta realidad, los autores creen que una posible consecuencia del ASI puede ser sufrir un desinvestimiento libidinal del cuerpo sexuado genitalmente que se manifiesta tanto en ofrecer su cuerpo para goce del partenaire como anestesiarlo al placer y al juego sexual subjetivante, 0 al contrario, que se produzca una inhibición marcada al juego sexual y a sentir placer en vínculos con pares (Franco et. al, 2013).

A pesar de que los dos usuarios del CRAm arribaron al espacio terapéutico manifestando en sus narrativas no sentir deseo sexual, no tener ningún tipo de atracción hacia terceros, no haber materializado prácticas sexuales vinculares y no vivenciaban su asexualidad como un problema, a medida que avanzaron en sus análisis, se presentaron diferencias de distinto alcance e intensidad entre ambos usuarios que terminaron posibilitando cambios en su sexualidad. Sin embargo, es una casuística pequeña, sería una imprudencia afirmar que toda persona que se autoidentifique como asexual tiene algún antecedente de ASI o que la base de la asexualidad está asociada a una experiencia traumática. Tampoco corresponde construir respuestas lineales, monocausales, plantear a la asexualidad como una consecuencia directa de un ASI. Concluir esto implicaría reconocer que la asexualidad no está dentro de lo esperable, sería un modo de patologizar toda 
representación de la asexualidad. Este posicionamiento podría ser leído como violento por aquellas personas que consideran que la asexualidad es su orientación o identidad sexual. Sin embargo, tenemos la responsabilidad de al menos hacernos preguntas. En este sentido: ¿cómo es el proceso de identificación con la asexualidad? ¿qué eventos, experiencias, hitos destacan como significativos las personas? ¿cómo se relacionan con su asexualidad? ¿qué cambios se producen a lo largo del tiempo en cuanto a las posibilidades de construcción y ejercicio de la sexualidad? ¿las experiencias de ASI pueden operar en la identificación con la asexualidad? ¿de qué manera?

\section{Conclusiones}

En este escrito se pretendió reflexionar desde una mirada crítica sobre la asexualidad. En este trabajo de aproximación a la temática no se buscó concluir si la asexualidad es una orientación sexual, una identidad sexual o un trastorno sexual. Simplemente se acercaron argumentos con rigurosidad científica que defienden o critican las tres posturas. Se propuso un diálogo interdisciplinario de saberes. Nuestra labor fue proponer una articulación teórica novedosa y actual a partir de la experiencia clínica desarrollada en el CRAm, que permitiera discutir nociones construidas como verdades incuestionables en el campo de la sexualidad humana, de hecho, probablemente, el alcance de este artículo trasciende a la propia asexualidad.

Un escenario posible es que en este momento ustedes se estén haciendo más preguntas que cuando iniciaron la lectura, si es así, humildemente consideramos que se cumplió con el objetivo principal de este escrito. A nosotros nos quedan resonando algunas interrogantes que nos desvelan: ¿es ético intervenir sobre la asexualidad de alguien? ¿se justifica en algún contexto? ¿en qué contexto se justifica? ¿desde dónde se debe intervenir para proteger los derechos humanos de las personas? ¿cómo sería un acompañamiento asertivo para estas personas? ¿cuál es el límite de la intervención? ¿se debe problematizar la categoría asexual? ¿es pertinente explorar su causa o simplemente se debe aceptar su existencia y no cuestionarla? ¿qué aportes pueden realizar las propias personas asexuales en todo esto?

Lejos de buscar patologizar a la asexualidad o instalar una verdad universal, este texto pretende oficiar como disparador para la reflexión, la crítica y la revisión de las distintas concepciones, enfoques y abordajes acerca de la asexualidad, con el fin de motivar la formulación de nuevas preguntas de investigación y promover un aumento de producción de conocimiento científico sobre el fenómeno porque actualmente ocupa un sitio periférico en la agenda de investigación sobre sexualidad humana en la región. Se debe insistir que se trata de una casuística muy pequeña, lo que se configura como una limitante, por lo cual, todas las conclusiones son parciales y no tienen la intención de ser aplicadas para todas las experiencias de asexualidad, más aún, si se considera el componente de cambio permanente de las distintas expresiones y manifestaciones de la sexualidad humana. Asimismo, se remarca una vez más, que en esta producción, se buscó visibilizar a las personas que se autoidentifican como asexuales, reconociendo sus experiencias, vivencias $y$ respetando su subjetividad. 


\section{Bibliografía}

Alvarez, L. (2010). La identidad "asexual". Gazeta de Antropología, 26 (2), 1-18.

American Psychiatric Association (APA). (2013). Guía de Consulta de los Criterios Diagnósticos del $D S M-V$. Arlington, Ustados Unidos: APA. Recuperado: https://www.eafit.edu.co/ninos/reddelaspreguntas/Documents/dsm-v-guia-consulta-manualdiagnostico-estadistico-trastornos-mentales.pdf

Asexual Visibility and Education Network (AVEN). (s/f). Overview. The Asexual Visibility \& Education Network. Recuperado: https://www.asexuality.org/?q=overview.html

Balta, S. y Moreno, P. (2015). Abuso sexual infantil: Cuestiones relevantes para su tratamiento en la justicia. Montevideo, Uruguay: UNICEF y Fiscalía General de la Nación Uruguay.

Barrientos, J. (2016). Situación social y legal de gays, lesbianas y personas transgénero y la discriminación contra estas poblaciones en América Latina. Revista Latinoamericana Sexualidad, Salud y Sociedad, 22, 331-354.

Berlinerblau, V. (2016). Abuso sexual contra niños, niñas y adolescentes: Una guía para tomar acciones y proteger sus derechos. Buenos Aires, Argentina: UNICEF.

Bogaert, A. (2012). Understanding Asexuality. Estados Unidos: Rowman \& Littlefield Publishers Inc. Recuperado:

https://books.google.com.uy/books?hl=es\&lr=\&id=3P2pVq9XIGsC\&oi=fnd\&pg=PR5\&dq=understa nding+asexuality+pdf\&ots=0 JM 84mG0\&sig=gXh654 8igaDPWulebYNTcLV984\#v=onepage\&q\&f =false

Brotto, L. y Yule, M. (2016). Asexuality: Sexual Orientation, Paraphilia, Sexual Dysfunction, or None of the Above? Archives of Sexual Behavior. 46, 619-627. Recuperado: https://med-fombrotto.sites.olt.ubc.ca/files/2017/04/Brotto-Yule-2017-Asexuality-Sexual-orientation-paraphiliasexual-dysfunction-or-none-of-the-above-.pdf

Byung-Chul, H. (2014). La agonía del eros. Barcelona, España: Herder Editorial, S. L.

Comisión Interamericana de Derechos Humanos (CIDH). (2015). Violencia contra Personas Lesbianas, Gay, Bisexuales, Trans e Intersex en América. Washington, Estados Unidos: CIDH.

Conley-Fonda, B. y Leisher, T. (2018). Asexuality: Sexual Health Does Not Require Sex. Sexual Addiction \& Compulsivity. $25 \quad$ (1), 6-11. Recuperado: https://www.tandfonline.com/doi/full/10.1080/10720162.2018.1475699

Connell, R.W. (2003). Masculinidades. CDMX, México: Universidad Nacional Autónoma de México.

De la Hermosa, M. y Usaola, C. (2018). Sexualidad, violencia sexual y salud mental. Revista de la Asociación Española de Neuropsiquiatría. 38 (134), 349-356. Recuperado: http://scielo.isciii.es/pdf/neuropsiq/v38n134/2340-2733-raen-38-134-0349.pdf

Revista del Laboratorio Iberoamericano para el Estudio Sociohistórico de las Sexualidades https://doi.org/10.46661/relies. 5334 
Echeburúa, E. y De Corral, P. (2006). Secuelas emocionales en víctimas de abuso sexual en la infancia. Cuad Med Forense. 43(44), 75-82. Recuperado: http://scielo.isciii.es/scielo.php?script=sci_arttext\&pid=S1135-76062006000100006

Foucault, M. (1998). Historia de la sexualidad. 1 La voluntad de saber. Madrid, España: Siglo XXI Editores.

Franco; A; Gomez, V; Topori, S; Germade, A; Santi, G; Woloski, G; Pañazola, N; Tkach, C; Pucci, M y Raschkovan, I. (2013). Abuso sexual infantil y el investimiento/desinvestimento libidinal del cuerpo sexuado genitalmente. Anuario de Investigaciones. 20, 49-63. Recuperado: https://www.redalyc.org/pdf/3691/369139949036.pdf

Freud, S. (1905). Tres ensayos de teoría sexual. Obras completas. Volumen VII. Buenos Aires, Argentina: Amorrortu Editores.

Garcia, L y Cerda, B. (2010). Violencia Sexual. CDMX, México: Instituto Nacional de Psiquiatria Ramon de la Fuente Muñiz.

Gelpi, G; Pascoll, N. y Egorov, D. (2019). Sexualidad y redes sociales online: Una experiencia educativa con adolescentes de Montevideo. Revista Iberoamericana De Educación, 80 (2), 61-80.

Giddens, A. (1995). La constitución de la sociedad. Bases para la teoría de la estructuración. Buenos Aires, Argentina: Amorrortu Editores.

Guasch, O. (2007). La crisis de la heterosexualidad. Barcelona, España: Editorial LAERTES.

Junqueira, R. (2013). Pedagogia do armário. A normatividade em ação. Revista Retratos da Escola, 7 (13), 481-498.

López, S. y Platero, L. (2019). Cuerpos marcados. Vidas que cuentan y políticas públicas. Barcelona, España: Bellaterra.

López, A. (Coord.). (2015). Adolescentes y sexualidad. Investigación, acciones y política pública en Uruguay (2005-2014). Montevideo, Uruguay: UNFPA.

López, P; Forrisi, F. y Gelpi, G. (2015). Salud y Diversidad Sexual: Guia para profesionales de la salud. Montevideo, Uruguay: UNFPA.

Louro, G. (2000). O corpo educado: Pedagogias da sexualidade. Belo Horizonte, Brasil: Autêntica.

Lozano, F. (2017). Violencia sexual: Recomendaciones para su abordaje desde los equipos de salud. Protocolo Institucional de Atención a Personas en Situación de Violencia Basada en Género y Generaciones de ASSE. Montevideo, Uruguay: MSP.

Marzano, M. (2015). Las paradojas de la pornografía contemporánea entre libertad, goce y estereotipos de sumisión. En C. Barzani (Comp), Actualidad de Erotismo y Pornografía. Buenos Aires, Argentina: Topía Editorial.

Revista del Laboratorio Iberoamericano para el Estudio Sociohistórico de las Sexualidades https://doi.org/10.46661/relies. 5334 
Quenan, N. y Samudio, G. (2013). Abuso sexual infantil: epidemiología y estudio de la conducta de los médicos pediatras pre y pos intervención educativa. Pediatría (Asunción), 40 (2), 125-131. Recuperado: $\quad$ http://scielo.iics.una.py/scielo.php?script=sci_abstract\&pid=S1683$\underline{98032013000200003 \& \operatorname{lng}=e n \& n r m=i s o \& t \operatorname{lng}=e s}$

Organización Mundial de la Salud (OMS). (2018). La salud sexual y su relación con la salud reproductiva: Un enfoque operativo, Ginebra, Suiza: OMS. Recuperado: https://www.who.int/reproductivehealth/publications/sexual health/sh-linkages-rh/es/

Organización Mundial de la Salud (OMS). (2019). Violencia. Ginebra, Suiza: OMS. Recuperado de: https://www.who.int/topics/violence/es/.

Organización Panamericana de la Salud (OPS). (2013). Comprender y abordar la violencia contra las mujeres. Washington D.C, Estados Unidos: OPS. Recuperado de: https://apps.who.int/iris/handle/10665/98821

Pereda, N. (2009). Consecuencias psicológicas iniciales del abuso sexual infantil. Papeles del Psicólogo. 30(2), 135-144. http://www.papelesdelpsicologo.es/pdf/1702.pdf

Perez, M. (2019). Violencia Epistémica: Reflexiones entre lo invisible y lo ignorable. Revista de estudios $y$ políticas de género, 1(1). 81-98. Recuperado: http://revistas.untref.edu.ar/index.php/ellugar/article/view/288/267

Radi, B. (2019). Políticas del conocimiento. Hacia una epistemología trans*. En M. López (Comp), Los mil pequeños sexos. Intervenciones críticas sobre políticas de género y sexualidades (pp.27-45). Buenos Aires, Argentina: Universidad Nacional de Tres de Febrero.

Ramos, S; Finzi, T. y Gualdoni, N. (2018). Abusos sexuales y embarazo forzado en la niñez y la adolescencia. Buenos Aires, Argentina: UNICEF. Recuperado: https://www.unicef.org/argentina/sites/unicef.org.argentina/files/2018-

11/AbusoSexual\%2BAnexoM\%C3\%A9dico Digital Nov2018.pdf

Ramos, V; Forrisi, F. y Gelpi, G. (2015). Nociones básicas sobre sexualidad, género y diversidad: Un lenguaje en común. En P. López, G. Gelpi, F. Forrisi (Ed), Salud y Diversidad Sexual: Guia para profesionales de la salud (pp. 15-47). Montevideo, Uruguay: UNFPA.

Sanchez. C; Morales, F; Carreño, J. y Cuenca, J. (2007). Disfunciones Sexuales Masculinas: su Asociación con el Malestar Psicológico y los Problemas Conyugales en una Población de la Ciudad de México. Psicología Iberoamericana. 15(1), 60-67. Recuperado: https://www.redalyc.org/pdf/1339/133915928008.pdf

Savin-Williams, R. (2009). La nueva adolescencia homosexual. Madrid, España: Ediciones Morata.

Scherrer, K. (2008). Coming to an Asexual Identity: Negotiating Identity, Negotiating Desire. Sexualities, 11(5), 621-641. Recuperado: https://www.ncbi.nlm.nih.gov/pmc/articles/PMC2893352/

Revista del Laboratorio Iberoamericano para el Estudio Sociohistórico de las Sexualidades https://doi.org/10.46661/relies. 5334 
Unidad de Igualdad de Género (UIG). (2017). Violencia Sexual. CDMX, México: Procuraduría General de la Nación.

Van Houdenhove, E; Enzlin, P y Gijs, L. (2017). A Positive Approach Toward Asexuality: Some First Steps, But Still a Long Way to Go. Archives of Sexual Behavior, 46 (3), 647-651.

Villanueva, I. (2013). El abuso sexual infantil: Perfil del abusador, la familia, el niño víctima y consecuencias psíquicas del abuso. Psicogente, 16(30), 451-470. Recuperado: https://www.redalyc.org/pdf/4975/497552364016.pdf

Weeks, J. (1988). La invención de la sexualidad. En J. Weeks (Ed), Sexualidad (pp. 21-46). CDMX, México: Paidós, PUEG, UNAM. 